\title{
Phylogenetic Relationship of Six Glycyrrhiza Species Based on rbcL Sequences and Chemical Constituents ${ }^{1)}$
}

\author{
Hiroaki Hayashi, ${ }^{*, a}$ Noriko Hosono, ${ }^{b}$ Mieko Kondo, ${ }^{b}$ Noboru Hiraoka, ${ }^{b}$ Yasumasa IKeshiro, ${ }^{b}$ \\ Makio Shibano, ${ }^{c}$ Genjiro Kusano, ${ }^{c}$ Hirobumi Yamamoto, ${ }^{d}$ Toshihiro TAnaka, ${ }^{a}$ and Kenichiro Inoue ${ }^{a}$ \\ Gifu Pharmaceutical University, ${ }^{a}$ 5-6-1 Mitahora-higashi, Gifu 502-8585, Japan., Niigata College of Pharmacy, ${ }^{b}$ 5-13-2 \\ Kamishinei-cho, Niigata 950-2081, Japan, Osaka University of Pharmaceutical Sciences, ${ }^{c} 4-20-1$ Nasahara, Takatsuki \\ 569-1094, Japan, and School of Pharmaceutical Sciences, Nagasaki University, ${ }^{d}$ I-14 Bunkyo-machi, Nagasaki \\ 852-8521, Japan. Received December 1, 1999; accepted January 14, 2000
}

\begin{abstract}
The nucleotide sequences of ribulose-1,5-bisphosphate carboxylase/oxygenase large subunit gene $(r b c L)$ of Glycyrrhiza glabra, G. uralensis, G. inflata, G. echinata, G. macedonica and G. pallidiflora have been determined to construct their phylogenetic tree. Based on these sequences, the six Glycyrrhiza species were divided into two groups: three, G. glabra, G. uralensis, and $G$. inflata, which produce glycyrrhizin as a major saponin, and the others, G. echinata, G. macedonica and G. pallidiflora, which produce macedonoside $\mathrm{C}$ as a major saponin. Among the three glycyrrhizin-producing species, only two nucleotide substitutions were observed between the $r b c L$ sequences of $G$. glabra and $G$. uralensis, and the sequence of $G$. uralensis was identical to that of $G$. inflata, indicating that G. uralensis and G. inflata are closely related. Among the three macedonoside C-producing species, only one nucleotide substitution was observed between those of $G$. echinata and $G$. macedonica, indicating that these two species are also closely related.
\end{abstract}

Key words Leguminosae; Glycyrrhiza; rbcL; glycyrrhizin; macedonoside C; phylogenetic tree

Licorice, roots and stolons of the Glycyrrhiza plants, $G$. glabra L., G. uralensis FisCH. and G. inflata BATAL., contain a large amount of glycyrrhizin (GL), which is a well recognized natural sweetener and pharmaceutical. ${ }^{2)}$ Some Glycyrrhiza species, such as G. echinata L., G. macedonica Borss. et ORPH and G. pallidiflora MAXIM., however, do not contain GL. ${ }^{3)}$ It is of interest to elucidate the phylogenetic relationship among these species from a viewpoint of the evolution of GL biosynthesis. Recently, nucleotide sequences of a chloroplast gene for the large subunit of ribulose-1,5-bisphosphate carboxylase/oxygenase $(r b c L)$ were used to produce phylogenetic trees of higher plants, ${ }^{4)}$ and comprehensive phylogenetic studies of legume $r b c L$ sequences were also reported ${ }^{5,6)}$ In a preliminary communication, ${ }^{1)}$ we determined the $r b c L$ sequences of three GL-producing and two GL-non-producing Glycyrrhiza species. In this paper, the phylogenetic relationship based on their chemical constituents and $r b c L$ sequences of six Glycyrrhiza species will be discussed.

\section{MATERIALS AND METHODS}

Plant Materials Seeds of G. glabra and G. echinata (collected in Turkey) were obtained from the medicinal plant garden of Kyoto University. Seeds of G. uralensis were obtained from the medicinal plant garden of Chiba University. Seeds of G. pallidiflora and G. macedonica were obtained from National Institute of Health Sciences, Japan, and a plant of G. inflata was obtained from Maruzen Pharmaceuticals Co., Ltd. All these species were cultivated in the medicinal plant garden of Niigata College of Pharmacy, and their leaves and roots were harvested for analysis.

Amplification and Sequencing of $r b c L$ Gene Total DNA was extracted from the fresh leaves by the cetyltrimethylammonium bromide (CTAB) DNA extraction method as reported previously. ${ }^{7}$ The 1374 -bp DNA fragment covering most of the $r b c L$ sequences was amplified by the polymerase chain reaction (PCR) using total DNA, Taq-DNA polymerase (Takara, Japan), and two primers, $5^{\prime}$-ATGTCACCACAAACAGAAACTAAAGC- $3^{\prime}$ and $5^{\prime}$-AGCAGCAGCTAATTCAGGACTCCA-3'. After initial denaturation (2 min at $95^{\circ} \mathrm{C}$, 25 cycles of $40 \mathrm{~s}$ at $95^{\circ} \mathrm{C}, 40 \mathrm{~s}$ at $55^{\circ} \mathrm{C}$ and $2 \mathrm{~min}$ at $72^{\circ} \mathrm{C}$ were performed on a thermocycler. The amplified fragment was purified by $1 \%$ agarose gel electrophoresis and Geneclean II kit (BIO 101 Inc., U.S.A.). The purified fragments were sequenced directly by the dideoxy chain termination method using an ALFred DNA sequencer (Pharmacia). Nine internal primers, located at every $300-400 \mathrm{bp}$ of both strands, were used for sequencing.

HPLC Analysis of Roots and Leaves Freeze-dried plant samples were ground with a mortar and pestle, and then $40 \mathrm{mg}$ of each powdered sample was extracted with $1 \mathrm{ml}$ of $80 \%$ methanol at $60^{\circ} \mathrm{C}$ for $6 \mathrm{~h}$. An aliquot $(20 \mu \mathrm{l})$ of the extract was analyzed by photodiode-array HPLC as reported previously. ${ }^{8)}$ Conditions of HPLC were as follows: column, Capcellpak C18 AG-120A ( $5 \mu \mathrm{m}, 4.6 \mathrm{~mm}$ i.d. $\times 250 \mathrm{~mm}$, Shiseido, Japan); solvent, $\mathrm{MeCN}-\mathrm{H}_{2} \mathrm{O}(1 \% \mathrm{AcOH})$ gradient of $15 \% \mathrm{MeCN}$ to $25 \% \mathrm{MeCN}$ in $15 \mathrm{~min}$, to $70 \% \mathrm{MeCN}$ in another $35 \mathrm{~min}$, then to $100 \%$ in $10 \mathrm{~min}$; flow rate, $0.9 \mathrm{ml} / \mathrm{min}$; column temp, $40^{\circ} \mathrm{C}$; detector, Shimadzu photodiode array SPDM-6A system (Shimadzu, Japan). Quantities of constituents were determined on the basis of the peak area of UV absorption at $254 \mathrm{~nm}$. Each constituent was identified by comparison of its retention time and UV spectrum with those of authentic sample.

\section{RESULTS AND DISCUSSION}

Comparison of Chemical Constituents in the Roots and Leaves of Six Glycyrrhiza Species As stated, the six Glycyrrhiza plants, i.e. G. glabra, G. uralensis, G. inflata, G. echinata, G. macedonica and G. pallidiflora, were cultivated in the medicinal plant garden of Niigata College of Pharmacy, and the roots and leaves were harvested for analysis of 

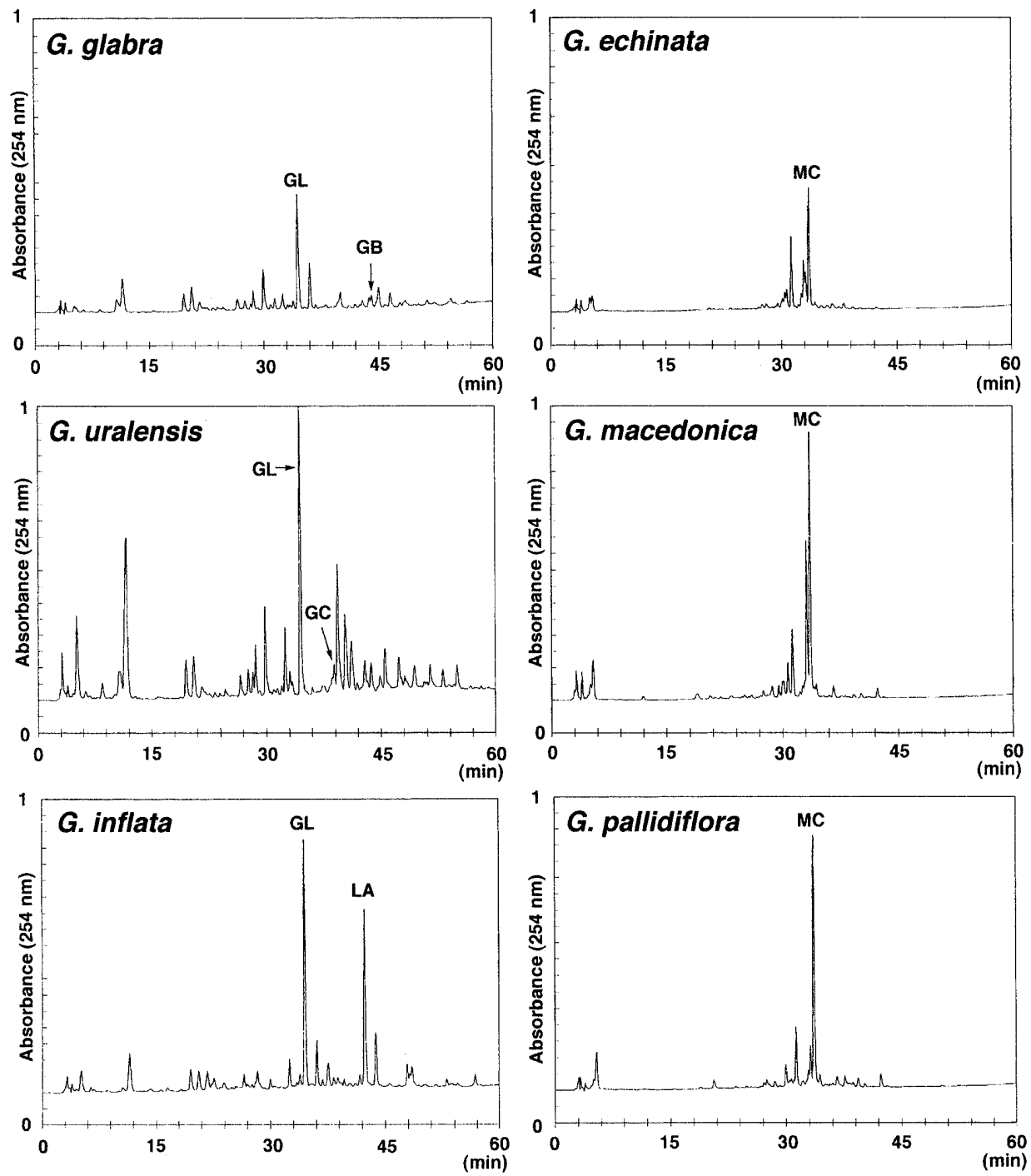

Fig. 1. HPLC Profiles of Methanol Extracts of the Roots of Six Glycyrrhiza Species

GB, glabridin; GC, glycycoumarin; GL, glycyrrhizin; LA, licochalcone A; MC, macedonoside C.

Table 1. Contents of Glycyrrhizin (GL), Macedonoside C (MC), Glabridin (GB), Glycycoumarin (GC) and Licochalcone A (LA) in Roots of Glycyrrhiza Plants

\begin{tabular}{lcccccc}
\hline \hline \multirow{2}{*}{ Species } & Diameter $(\mathrm{mm})$ & \multicolumn{6}{c}{ Contents (\% of dry weight) of } \\
\cline { 3 - 7 } & & GL & MC & GB & GC & LA \\
\hline G. glabra & 10.5 & 1.26 & n.d. & 0.11 & n.d. & n.d. \\
G. uralensis & 9.2 & 3.27 & n.d. & n.d. & 0.20 & n.d. \\
G. inflata & 6.4 & 2.49 & n.d. & n.d. & n.d. & 0.64 \\
G. echinata & 7.3 & n.d. & 1.19 & n.d. & n.d. & n.d. \\
G. macedonica & 6.7 & n.d. & 2.58 & n.d. & n.d. & n.d. \\
G. pallidiflora & 9.1 & n.d. & 2.33 & n.d. & n.d. & n.d. \\
\hline
\end{tabular}

chemical constituents and $r b c L$ sequence. Figure 1 shows HPLC profiles of methanol extracts of the thickening roots of the six species and Table 1 shows the contents of two saponins, glycyrrhizin (GL) and macedonoside $\mathrm{C}(\mathrm{MC})$, and three flavonoids, glabridin (GB), glycycoumarin (GC) and licochalcone A (LC), known to be species-specific for $G$. glabra, G. uralensis and G. inflata, respectively, in the thickening roots. ${ }^{9,10)}$ The structures of these compounds are shown in Fig. 2. In the thickening roots of G. glabra, G. uralensis

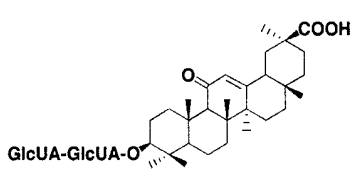

Glycyrrhizin (GL)
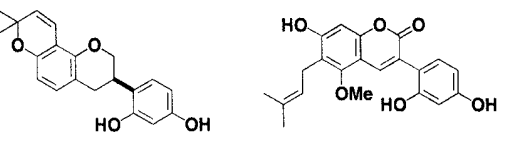

Macedonoside C (MC)

Glabridin (GB)

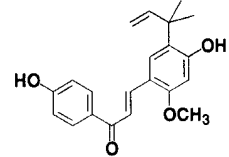

Licochalcone A (LA)
Fig. 2. Structures of Saponins and Flavonoids in the Roots of Glycyrrhiza Plants

and G. inflata, GL was detected in common, and $\mathrm{GB}, \mathrm{GC}$ and LA were specifically detected in the thickening roots of these three species, respectively. In the remaining three species, $G$. echinata, G. macedonica and G. pallidiflora, the HPLC profiles were closely similar, and MC, which was isolated for the first time from G. macedonica, ${ }^{3)}$ was detected instead of GL as the main saponin.

Next, HPLC analysis was made of methanol extracts of the leaves of the six Glycyrrhiza species and the HPLC profiles 

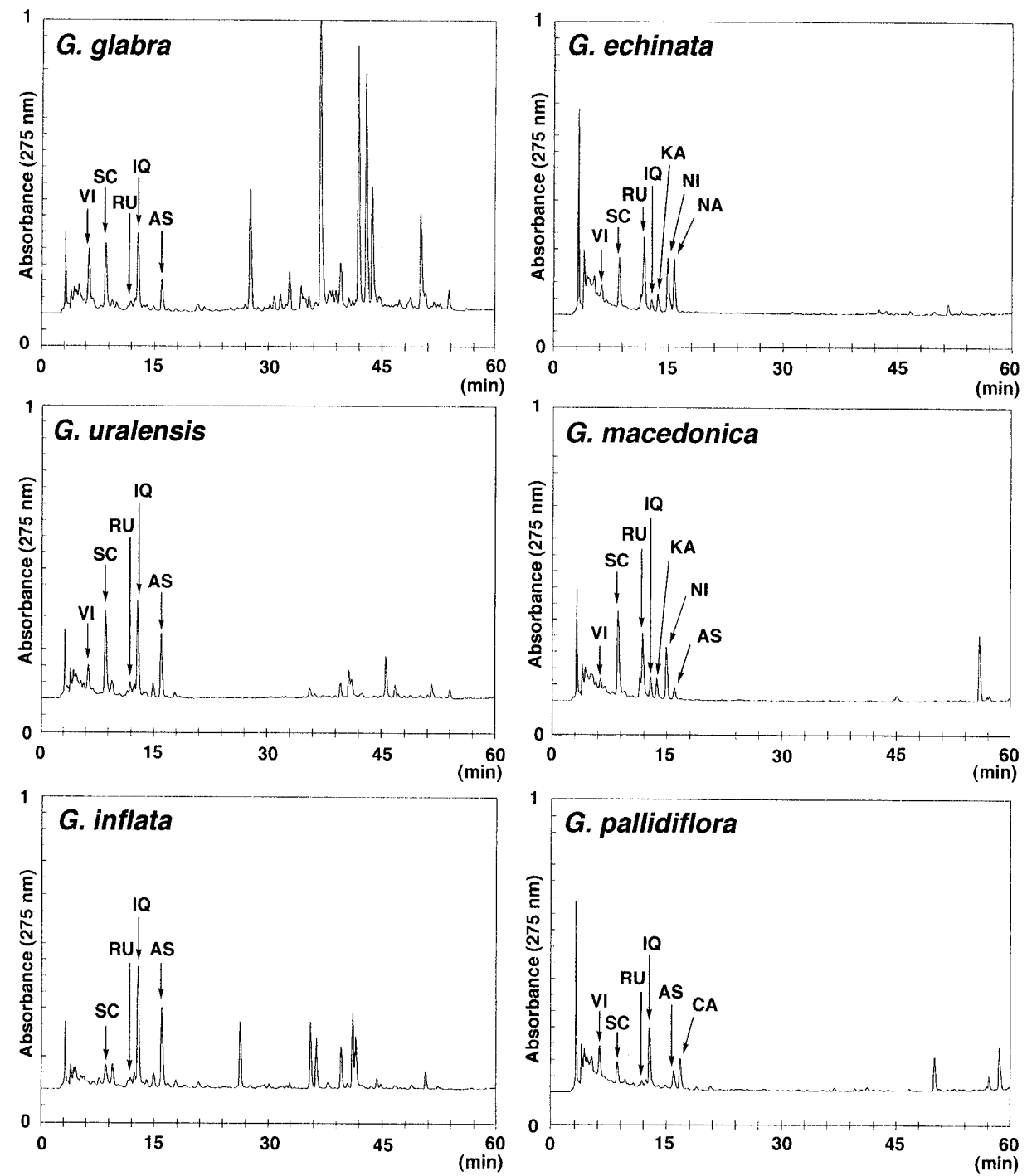

Fig. 3. HPLC Profiles of Methanol Extracts of the Leaves of the Six Glycyrrhiza Species

AS, astragalin; CA, cacticin; IQ, isoquercitrin; KA, kaempferol-3-o-rhamnosylgalactoside; NA, narcissin; NI, nicotiflorin; RU, rutin; SC, schaftoside; VI, vicenin 2.

are shown in Fig. 3. The structures of flavonoid glycosides identified in this experiment are shown in Fig. 4. In the leaves of the three GL-producing species, which contained isoquercitrin (IQ) and astragalin (AS) as major flavonoid glycosides, the patterns of flavonoid glycoside constituents were similar, whereas in those of the three MC-producing species, the species-specific distribution of flavonoid glycosides was observed. Although the profiles of flavonoid glycosides were similar in the leaves of G. echinata and G. macedonica, the leaves of G. echinata contained narcissin (NA) instead of the AS present in G. macedonica. Both G. echinata and $G$. macedonica contained rutin (RU) as a major flavonoid glycoside, whereas the major flavonoid glycoside in G. pallidiftora was IQ. The HPLC profile of flavonoid glycoside in the leaves of G. pallidiflora, a MC-producing species, was different from those of the other two MC-producing species, $G$. echinata and G. macedonica: in G. pallidiflora, cacticin (CA) was detected as a species-specific flavonoid glycoside. These HPLC profiles of the leaves of the three MC-producing species were identical to those of previous reports. ${ }^{3,11)}$ Species-specific peaks were also observed at retention times of from 30 to $60 \mathrm{~min}$ in the HPLC profiles of all six species, respectively. Some of them have been identified as free

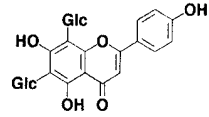

Vicenin $2(\mathrm{VI})$

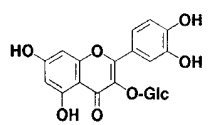

Isoquercitrin (IQ)

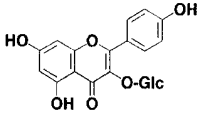

Astragalin (AS)

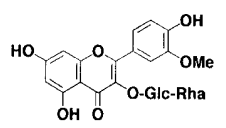

Narcissin (NA)

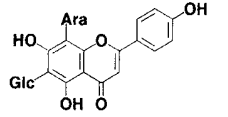

Schaftoside (SC)

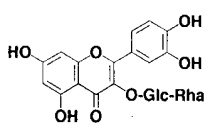

Rutin (RU)

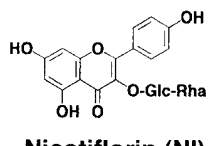

Nicotiflorin (NI) Kaempferol-3-O-rhamnosyl-<smiles></smilesgalactoside (KA)
Fig. 4. Structures of Flavonoid Glycosides in the Leaves of Glycyrrhiza Plants 
Table 2. Pairwise Genetic Distances between the Six Glycyrrhiza Species and Abrus precatorius Based on 1324 Nucleotides (without Primers) of the rbcL Gene

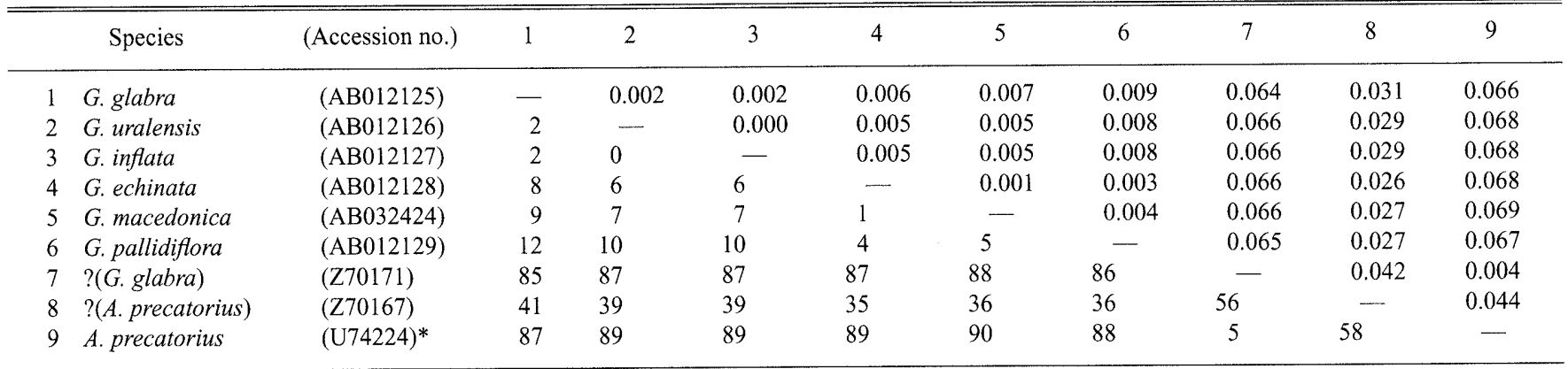

* 1310 nucleotides were used for comparison with the other sequences.

Below diagonal: number of nucleotide substitutions; above diagonal: proportion of nucleotide positions differing between taxa $(1.0=100 \%)$

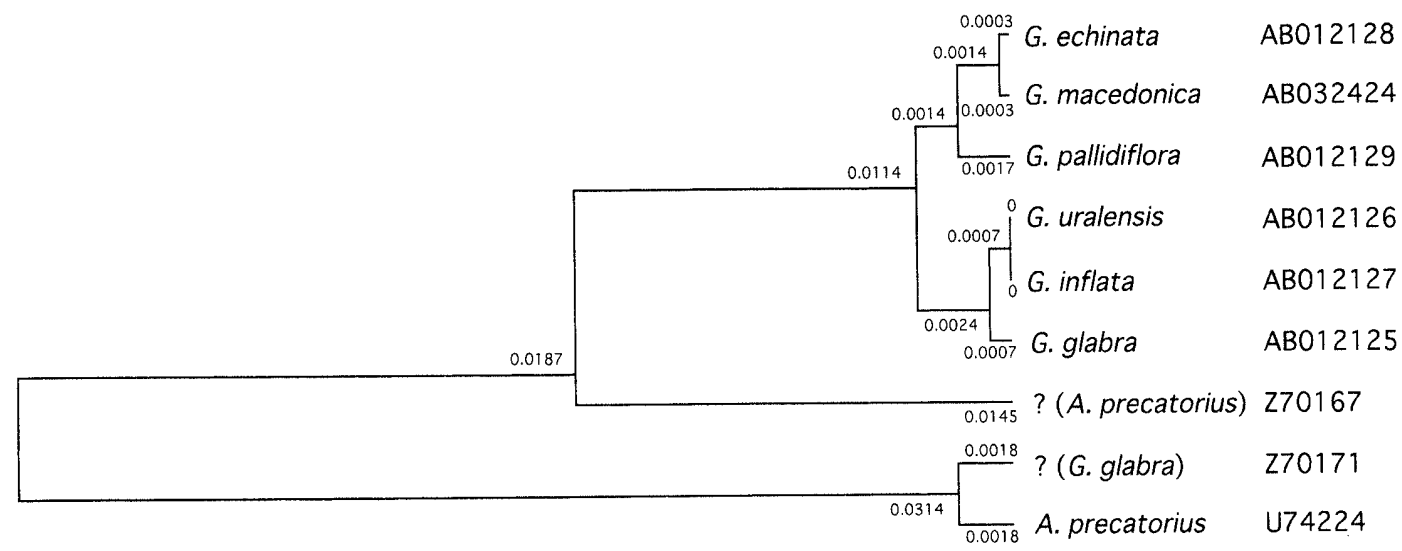

Fig. 5. Phylogenetic Tree Constructed from the Nucleotide Sequences of $r b c L$ Genes of the Six Glycyrrhiza Species by the UPGMA method of Nei The phylogenetic tree was constructed by Genetyx software, Software Development, Japan. The database accession numbers are shown on the right.

flavonoids and isoflavonoids, and the variation of these constituents was also reported in G. glabra. ${ }^{11,12)}$

Phylogenetic Relationship of Six Glycyrrhiza Species Based on $r b c L$ Sequences Total DNA was extracted from the fresh leaves of the six Glycyrrhiza species as reported previously, ${ }^{7)}$ and the 1374-bp DNA fragment covering most of the $r b c L$ sequence was amplified by PCR and sequenced. ${ }^{13)}$ Surprisingly, $r b c L$ sequences of all six species determined in the present study were different from that of $G$. glabra (accession No. Z70171) reported by Häss and Wink. ${ }^{5}$ Doyle et al. ${ }^{6)}$ reported that the $r b c L$ sequence of Abrus precatorius L. (accession No. U74224) was very similar to that of G. glabra (accession No. Z70171) by Häss and Wink, ${ }^{5)}$ and different from that of $A$. precatorius (accession No. Z70167) by Häss and Wink. ${ }^{5)}$ These results are attributed to a simple switch of $G$. glabra and A. precatorius sequences. However, the sequence of accession No. Z70167 is also different from that of G. glabra obtained in the present study, which is very similar to $r b c L$ sequences of the other five $G l y$ cyrrhiza species. This suggests that the original plant for accession No. Z70167 is not G. glabra, but is somewhat related to the Glycyrrhiza species. Table 2 shows the pairwise genetic distances based on the $r b c L$ sequences. A phylogenetic tree (Fig. 5) was constructed from the nucleotide sequences of the $r b c L$ gene using the unweighted pair-group method using arithmetic averages (UPGMA method) of Nei ${ }^{14)}$ (Genetyx software, Software Development, Japan). The six Glycyrrhiza species were divided into two groups: the three GL-producing species, G. glabra, G. uralensis, and $G$. inflata, and the three MC-producing species, G. echinata, $G$. macedonica and G. pallidiflora. The result coincides with a phylogenetic tree suggested by RFLP analyses of four Glycyrrhiza species, i.e. G. glabra, G. uralensis, G. echinata and G. pallidiflora. ${ }^{15)}$ It is noteworthy that the $r b c L$ sequence of $G$. uralensis was identical to that of $G$. inflata, indicating that $G$. uralensis and $G$. inflata are closely related, and that only two nucleotide substitutions were observed between $G$. glabra and G. uralensis. The $r b c L$ sequences were also similar among the GL-non-producing species, which contain MC in the roots. Only one nucleotide substitution was observed between G. echinata and G. macedonica, and their HPLC profiles of the thickening roots and leaves were also very similar. These results indicate that the two species are closely related. The present study indicates that the phylogenetic relationship deduced from their $r b c L$ sequence is in accordance with the similarity in their chemical constituents. The $r b c L$ sequence is a good marker to identify the GL-non-producing Glycyrrhiza species. It is difficult, however, to distinguish the various strains of GL-producing species by this sequence since it is very similar in all of them.

Acknowledgments The authors would like to thank Mr. Y. Tamura (Maruzen Pharmaceuticals), Dr. E. Sakai (National Institute of Health Sciences), Dr. F. Ikegami (Chiba University), and Prof. G. Honda (Kyoto University) for providing the Glycyrrhiza plants. They also would like to thank Mr. K. Kondo (Tsumura Central Research Laboratories) for his helpful advice. 


\section{REFERENCES AND NOTES}

1) Preliminary communication on this subject: Hayashi H., Hosono N., Kondo M., Hiraoka N., Ikeshiro Y., Biol. Pharm. Bull., 21, 782-783 (1998).

2) Gibson M. R., J. Nat. Prod., 41, 348-354 (1978).

3) Shibano M., Nukui H., Kita S., Kusano G., Shibata T., Watanabe H., Ohashi H., Natural Med., 53, 166-172 (1999).

4) Chase M. W., Soltis D. E., Olmstead R. G., Morgan D., Les D. H., Mishler B. D., Duvall M. R., Price R. A., Hills H. E., Qiu Y.-L., Kron K. A., Rettig J. H., Conti E., Palmer J. D., Manhart J. R., Sytsma K. J., Michaels H. J., Kress W. J., Karol K. G., Clark W. D., Hedren M., Gaut B. S., Jansen R. K., Kim K.-J., Wimpee C. F., Smith J. F., Furnier G. R., Strauss S. H., Xiang Q.-Y., Plunkett G. M., Soltis P. S., Swensen S. M., Williams S. E., Gadek P. A., Quinn C. J., Eguiarte L. E., Golenberg E., Learn G. H., Graham S. W., Barrett S. C. H., Dayanandan S., Albert V. A., Ann. Missouri Bot. Gard., 80, 528-580 (1993).

5) Häss E., Wink M., Bot. Acta, 108, 149-162 (1995).

6) Doyle J. J., Doyle J. L., Ballenger J. A., Dickson E. E., Kajita T., Ohashi H., Amer. J. Bot., 84, 541-554 (1997).

7) Murray M. G., Thompson W. F., Nucleic Acids Res., 8, 4321-4325
(1980).

8) Hayashi H., Hiraoka N., Ikeshiro Y., Yamamoto H., Plant Science, 116, 233-238 (1996).

9) Shibata S., Saitoh T., J. Indian Chem. Soc., 55, 1184-1191 (1978).

10) Hatano T., Fukuda T., Liu Y. Z., Noro T., Okuda T., Yakugaku Zasshi, 111, 311-321 (1991).

11) Shibano M., Matsumoto Y., Kusano G., Shibata T., Natural Med., 50 273-283 (1996)

12) Hayashi H., Yasuma M., Hiraoka N., Ikeshiro Y., Yamamoto H., Yesilada E., Sezik E., Honda G., Tabata M., Phytochemistry, 42, 701-704 (1996).

13) The nucleotide sequence data reported in this paper are deposited in the DDBJ, EMBL, GenBank with the accession numbers: AB012125 (G. glabra), AB012126 ( $G$. uralensis), $\mathrm{AB} 012127$ (G. inflata), AB012128 (G. echinata), AB012129 (G. pallidiflora), and AB032424 (G. macedonica).

14) Nei M., "Molecular Evolutionary Genetics," Columbia University Press, New York, 1987, pp. 293-298.

15) Yamazaki M., Sato A., Shimomura K., Saito K., Murakoshi I., Biol. Pharm. Bull., 17, 1529-1531 (1994). 